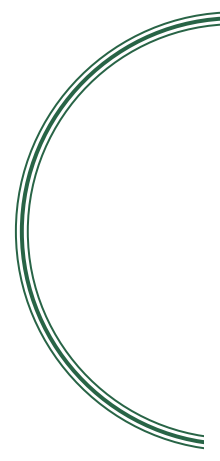

\title{
Buried Treasure: Unlocking the Secrets of Medicinal Mushrooms
}

\author{
Emma L. Walton
}

Staff Writer at the Biomedical Journal, Dronningens gate 56, Trondheim, Norway

In this issue of the Biomedical Journal, we investigate the potential of plants and fungi as a source of beneficial molecules for human health. We explore the weird and wonderful world of the mushroom and examine how Western medicine still has a lot to learn from Eastern practices dating back thousands of years. We also discuss a study further supporting claims that flaxseed, the plant kingdom's richest source of omega-3 fatty acids, can have lipid-lowering and fat-busting properties in the right physiological context. Finally, this issue also includes several validation studies of medical procedures or devices that define optimal conditions for their use in Asian populations. (Biomed J 2014;37:339-342)

Key words: $\beta$-glucans, cholesterol, flaxseed, immunomodulation, medicinal mushrooms, omega-3 fatty acid

\section{SPOTLIGHT ON REVIEWS - Bridging \\ The Gap between Eastern and Western Medicine with Medicinal Mushrooms}

$\mathrm{H}$ ailed by the ancient Egyptians and Chinese as the key to longevity, mushrooms have been used in traditional medicine for thousands of years. In this issue of the Biomedical Journal, Solomon Wasser $^{[1]}$ and Russell Paterson and Nelson Lima ${ }^{[2]}$ discuss the place of medicinal mushrooms (MMs) in today's world of evidence-based medicine and some of the challenges faced in unlocking the secrets of one of nature's greatest treasure troves.

Neither a plant nor an animal, fungi occupy one of the largest kingdoms in the tree of life thought to contain around 1.5 million species ${ }^{[3]}$ Mushrooms have been harvested by humans as a source of food and medicine for centuries. Particularly revered in traditional Chinese medicine are Reishi (Ganoderma lucidum) and Shiitake (Lentinus edodes) mushrooms [Figure 1]. Many controlled studies have since investigated the long list of medicinal actions thought to be associated with extracts of these and other mushrooms, including antitumor, immunomodulatory, antioxidant, antiviral, antibacterial, and hepatoprotective effects. Much evidence now supports the practices of our ancestors. For example, in a groundbreaking study published last year, Taiwanese scientists showed that mice immunized with a type of polysaccharide found in Reishi mushrooms produce antibodies that recognize tumor cell antigens. ${ }^{[4]}$

Mushrooms are a rich source of these polysaccharides, and many have been shown to stimulate host immune responses. Indeed, the effects of polysaccharides on the immune system are by far the most exploited attribute of MMs. Some of the most potent immunostimulatory molecules derived from mushrooms are the $\beta$-glucans, which activate many types of immune cells and stimulate cytokine responses. ${ }^{[5,6]}$ Studies in rodents and humans suggest that polysaccharides derived from MMs can stimulate the immune system to attack malignant cells, ${ }^{[7,8]}$ and several of these compounds are now used in Japan, Korea, and China as adjuncts to radio- and chemotherapy.

Western medicine, however, has been reluctant

DOI: $10.4103 / 2319-4170.146538$ 


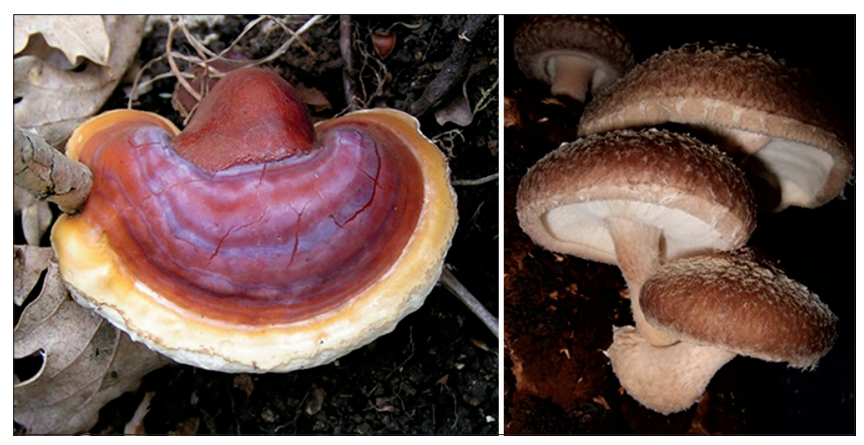

Figure 1: Reishi mushrooms (left) and their more palatable relatives the Shiitake mushrooms (right) have been used in traditional Eastern medicine for thousands of years. Images courtesy of Wikipedia.

to adopt these practices. The administration of complex mixtures of molecules of unknown concentrations is difficult to reconcile with current pharmaceutical practices involving highly purified compounds. The active ingredients may be unknown, making mushroom extracts very difficult to patent. Moreover, mushroom-derived polysaccharides are complex molecules that cannot be synthesized, so mass production of these compounds would require timely and costly extraction processes. As a result, many research efforts have focused on low molecular weight compounds, such as cordycepin, which is a cytotoxic nucleoside analog that inhibits cell proliferation. ${ }^{[9]}$

Despite these hurdles, MMs have already given us several drugs that have revolutionized modern medicine, including penicillin, statins, cyclosporine, and avermectin. Yet, according to recent estimates, only around $10 \%$ of species are known to mankind. ${ }^{[10]}$ Wasser describes MMs as "the greatest unclaimed gift of nature." Maybe the best is yet to come.

\section{SPOTLIGHT ON ORIGINAL ARTICLES - Fighting Flab with Flaxseed}

Cardiovascular diseases (CVDs) are the leading cause of death worldwide, ${ }^{[11]}$ and research on dietary supplements that might lower the risk of CVD is booming. In this issue of the Biomedical Journal, Saxena and Katare investigate the effects of flaxseed in individuals with dyslipidemia. ${ }^{[12]}$ Their findings appear to confirm the fat-busting and lipid-lowering properties of this much talked about plant.

You have probably already heard about the potential benefits of incorporating flaxseed into the diet. Flaxseed is the richest source of the omega- 3 fatty acid, $\alpha$-linolenic acid (ALA), which is thought to protect against CVDs. ${ }^{[13]}$ It also contains a high amount of phytohormone lignans, which have lipid-lowering and antioxidant properties. ${ }^{[14]}$ Top these two attributes off with a high dose of dietary fiber, and it is no wonder that flaxseed merits its classification as a "functional food." "[15]

In their latest study, Saxena and Katare examined how flaxseed affects biological variables related to
CVD in individuals with dyslipidemia. They divided 50 participants with similar diet and activity levels into two groups: an experimental group which took $30 \mathrm{~g}$ of flaxseed powder every day for 3 months and a control group which took no dietary supplement at all. At the end of the study, the experimental group lost on average one point on the body mass index (BMI) scale, whereas weight in the control group remained the same. Furthermore, flaxseed produced some astounding changes to the lipid profile of the experimental group: total cholesterol levels dropped by $16 \%$, triglycerides by $14 \%$ and low-density lipopro tein (LDL) by $20 \%$. Participants taking flaxseed also showed a small but significant reduction in blood pressure, but no changes occurred in the control group.

Thus, flaxseed helped participants to shed pounds and lower their cholesterol levels. It has to be pointed out that the control group was not administered any supplement, so the placebo effect cannot be excluded. Nonetheless, these findings are consistent with previous placebo-controlled studies showing that flaxseed supplementation lowers blood pressure. ${ }^{[16,17]}$ A meta-analysis of studies investigating the effects of flaxseed on blood lipid profile concluded that flaxseed significantly reduces circulating total and LDL-cholesterol concentrations, but the changes were dependent on sex and initial lipid profiles of the participants. ${ }^{[18]}$

In today's society of quick fixes, we should be cautious of health food companies selling miracle pills. The obesity epidemic cannot be solved by sprinkling powder onto your cereal in the morning. But if incorporated into a healthy diet and active lifestyle, there is no denying that flaxseed is a little seed with big potential.

\section{ALSO IN THIS ISSUE: REVIEWS - Mistaken Identity: True Function of Zucchini Revealed by Crystal Structures}

Zucchini is a member of the phospholipase D family of enzymes that make the lipid signaling molecule phosphatidic acid. Zucchini is required for the production of PIWI-interacting RNAs in germ cells, suggesting that lipid signaling is involved in this process. Here, Nureki discusses three recent crystal structures that shed light on zucchini and reveal its true function as a single-strand ribonuclease and not as a phosphodiesterase as previously thought. ${ }^{[19]}$

\section{ORIGINAL ARTICLES - Small, Dense and Deadly}

Low-density lipoprotein cholesterol (LDL-C) is a 
known risk factor for coronary artery disease (CAD) and decision to treat is often based on LDL-C levels. Huang and colleagues show that levels of the closely related small dense LCL (sdLCL) are also elevated in diabetic patients with CAD. ${ }^{[20]}$ Thus, assessment of both sdLCL and LDL-C levels is a better approach to determine the risk of CAD in this population.

\section{High score for the CoaguChek XS Plus System in Taiwanese Patients}

The CoaguChek XS Plus system is a portable device that provides an instant measure of prothrombin time and international normalized ratio (INR). Patients receiving the anticoagulant warfarin require frequent INR monitoring to avoid adverse reactions that may even cause death. Here, Fu et al. ${ }^{[21]}$ investigate the clinical and genetic factors that could affect the reliability of the CoaguChek XS Plus system in Taiwanese patients, and show that the results of this device are consistent with those of laboratory methods.

\section{Automated Renal Biopsies Deemed Safe and Reliable in Pediatric Setting}

Yu and colleagues assess the complications associated with percutaneous ultrasound-guided renal biopsy in asymptomatic children with microscopic hematuria. ${ }^{[22]}$ Their findings reaffirm the safety and reliability of this method for early diagnosis and also provide insight into the leading causes of kidney disease among Taiwanese children.

\section{Study Identifies Best Method for Estimating Kidney Function in Asian Populations}

Three equations are available to determine glomerular filtration rate (GFR), which is considered the best measure of kidney function. Kuo et al..$^{[23]}$ report the first study to investigate the suitability of these measures in a large Asian population (over 30,000 individuals). They identify the best measure of GFR among Taiwanese and warn against the use of the Cockcroft-Gault equation, which may grossly overestimate the prevalence of chronic kidney disease in this population.

\section{Taiwanese Patients Spring Back into Step Following Partial Knee Replacement}

Wong et al. ${ }^{[24]}$ investigate the functional outcome and patient satisfaction following uni-knee arthoplasty, which is an increasingly popular treatment option among qualifying patients requiring knee replacement surgery. An impressive $98 \%$ of the 48 patients surveyed were satisfied with the operation when asked 2-6 years later. Approximately three quarters were able to perform normal daily activities in Eastern societies, such as kneeling for religious services.

\section{BRIEF COMMUNICATION - Detecting Methicillin-Resistant Staphylococcus aureus in Resource-Limited Settings}

When resources are limited, conventional antibiotic susceptibility testing must replace gold standard molecular methods for the detection of methicillin-resistant Staphylococcus aureus (MRSA). However, the reliability of phenotypic tests depends on culture conditions. Kali et al..$^{[25]}$ assess the performance of several laboratory methods and show that oxacillin screen agar is the best alternative to PCR for the detection of MRSA.

\section{CORRESPONDENCE - How is Impact Factor Impacting our Research?}

A colleague has a paper published in a journal you have not heard of. The first question that comes to mind is: What is the impact factor? In this insightful commentary, Seema Rawat explains how the impact factor is calculated and points out many of the problems with this now widely accepted measure of influence. ${ }^{[26]}$

\section{LETTER - Comment on Farhadi et al.}

Kurt and colleagues comment on a study published in a previous issue of the Biomedical Journal showing that morning serum melatonin levels are low in individuals with multiple sclerosis, ${ }^{[27,28]}$ and suggest that several methodological issues should be addressed before such a conclusion can be drawn.

\section{REFERENCES}

1. Wasser SP. Medicinal mushroom science: Current perspectives, evidences, advances, and challenges. Biomed J 2014;37:345-56.

2. Paterson RR, Lima N. Biomedical effects of mushrooms with emphasis on pure compounds. Biomed J 2014;37:357-68.

3. Hawksworth DL. Mushrooms: The extent of the unexplored potential. Int J Med Mushrooms 2001;3:333-40.

4. Liao SF, Liang CH, Ho MY, Hsu TL, Tsai TI, Hsieh YS, et al. Immunization of fucose-containing polysaccharides from Reishi mushroom induces antibodies to tumor-associated Globo H-series epitopes. Proc Natl Acad Sci USA 2013;110:13809-14.

5. Takimoto H, Wakita D, Kawaguchi K, Kumazawa Y. Potentiation of cytotoxic activity in naive and tumor-bearing mice by oral administration of hot-water extracts from Agaricus brazei fruiting bodies. Biol Pharm Bull 2004;27:404-6.

6. Vetvicka V, Vashishta A, Saraswat-Ohri S, Vetvickova J. Immunological effects of yeast- and mushroom-derived betaglucans. 
J Med Food 2008;11:615-22.

7. Kurashige S, Akuzawa Y, Endo F. Effects of Lentinus edodes, Grifola frondosa and Pleurotus ostreatus administration on cancer outbreak, and activities of macrophages and lymphocytes in mice treated with a carcinogen, N-butyl-N-butanolnitrosoamine. Immunopharmacol Immunotoxicol 1997;19:175-83.

8. Ahn WS, Kim DJ, Chae GT, Lee JM, Bae SM, Sin JI, et al. Natural killer cell activity and quality ofWWW life were improved by consumption of a mushroom extract, Agaricus blazei Murill Kyowa, in gynecological cancer patients undergoing chemotherapy. Int J Gynecol Cancer 2004;14:589-94.

9. Tuli HS, Sharma AK, Sandhu SS, Kashyap D. Cordycepin: A bioactive metabolite with therapeutic potential. Life Sci 2013;93:863-9.

10. Hawksworth DL. Global species number of fungi: Are tropical studies and molecular approaches contributing to a more robust estimate? Biodivers Conserv 2012;21:242533.

11. Available from: http://www.who.int/mediacentre/factsheets/fs317/ en/[Last accessed on 2014 Nov 23].

12. Saxena S, Katare C. Evaluation of flaxseed formulation as a potential therapeutic agent in mitigation of dyslipidemia. Biomed $\mathrm{J}$ 2014;37:386-90.

13. de Lorgeril M, Salen P. Alpha-linolenic acid and coronary heart disease. Nutr Metab Cardiovasc Dis 2004;14:162-9.

14. Vanharanta M, Voutilainen S, Nurmi T, Kaikkonen J, Roberts LJ, Morrow JD, et al. Association between low serum enterolactone and increased plasma F2-isoprostanes, a measure of lipid peroxidation. Atherosclerosis 2002;160:465-9.

15. Giada Mde L. Food applications for flaxseed and its components: Products and processing. Recent Pat Food Nutr Agric 2010;2:181-6.

16. Cornish SM, Chilibeck PD, Paus-Jennsen L, Biem HJ, Khozani T, Senanayake $\mathrm{V}$, et al. A randomized controlled trial of the effects of flaxseed lignan complex on metabolic syndrome composite score and bone mineral in older adults. Appl Physiol Nutr Metab 2009;34:89-98.

17. Paschos GK, Magkos F, Panagiotakos DB, Votteas V, Zampelas A.
Dietary supplementation with flaxseed oil lowers blood pressure in dyslipidaemic patients. Eur J Clin Nutr 2007;61:1201-6.

18. Pan A, Yu D, Demark-Wahnefried W, Franco OH, Lin X. Meta-analysis of the effects of flaxseed interventions on blood lipids. Am J Clin Nutr 2009; 90:288-97.

19. Nureki O. Is zucchini a phosphodiesterase or a ribonuclease? Biomed J 2014;37:369-74.

20. Huang YC, Chang PY, Hwang JS, Ning HC. Association of small dense low-density lipoprotein cholesterol in type 2 diabetics with coronary artery disease. Biomed J 2014;37:375-9.

21. Fu CH, Chen WT, Chang PY, Michael Lee MT, Wen MS Revalidation of CoaguChek XS Plus System for INR monitoring in Taiwanese patients: Effects of clinical and genetic factors. Biomed J 2014;37:380-5.

22. Yu MC, Lee F, Huang WH, Aseuh S. Percutaneous ultrasound-guided renal biopsy in children: The need for renal biopsy in pediatric patients with persistent asymptomatic microscopic hematuria. Biomed J 2014;37:391-7.

23. Kuo CF, Yu KH, Shen YM, See LC. The Chinese version of the modification of diet in renal disease equation is a superior screening tool for chronic kidney disease among middle-aged Taiwanese than the original MDRD and Cockcroft-Gault equations. Biomed J 2014;37:398-405.

24. Wong T, Wang CJ, Wang JW, Ko JY. Functional outcomes of uni-knee arthroplasty for medial compartment knee arthropathy in Asian patients. Biomed J 2014;37:406-10.

25. Kali A, Stephen S, Umadevi S. Laboratory evaluation of phenotypic detection methods of methicillin-resistant Staphylococcus aureus. Biomed J 2014;37:411-4.

26. Rawat S. How is impact factor impacting our research? Biomed J 2014;37:415-6.

27. Kurt YS, Agilli M, Aydin FN. Morning Serum Melatonin Values in Multiple Sclerosis: A Biochemical Evaluation. Biomed J 2014;37:417.

28. Farhadi N, Oryan S, Nabiuni M. Serum levels of melatonin and cytokines in multiple sclerosis. Biomed J 2014;37:90-2. 